\title{
Analysis of Resonance Absorption in Multilayered Thin-Film Bi-Grating
}

\author{
Taikei Suyama \\ Department of Electrical and Computer Engineering, National Institute of Technology, Akashi College, \\ Akashi, Hyogo, Japan \\ Email: suyama@akashi.ac.jp
}

How to cite this paper: Suyama, T. (2019) Analysis of Resonance Absorption in Multilayered Thin-Film Bi-Grating. Journal of Modern Physics, 10, 1606-1614. https://doi.org/10.4236/jmp.2019.1013106

Received: October 29, 2019

Accepted: November 24, 2019

Published: November 27, 2019

Copyright (C) 2019 by author(s) and Scientific Research Publishing Inc. This work is licensed under the Creative Commons Attribution International License (CC BY 4.0).

http://creativecommons.org/licenses/by/4.0/

\begin{abstract}
The resonance absorption of a multilayered bi-grating which consists of thin-film corrugated periodically in two directions is investigated. The absorption in a multilayered thin-film bi-grating has been of considerable interest since we can expect more complex behaviors in the absorption phenomen by virtue of the presence of double periodicity and multilayer structure. In solving the problem, we employed a computational technique based on modal expansion. Taking a sandwiched structure $/ \mathrm{Ag} / \mathrm{SiO}_{2} / \mathrm{Ag} /$ for an example, we observed: 1) excitation of a single-interface surface plasmon mode at the lit surface of the $1^{\text {st }} \mathrm{Ag}$ layer with strong field enhancement for thick enough $\mathrm{Ag}$ layer case; 2) excitation of coupled short-range or long-range surface plasmon modes at each surface between vacuum and Ag layers with strong field enhancements for thin enough $\mathrm{Ag}$ layer cases no matter with the thickness of $\mathrm{SiO}_{2}$ layers; 3) enhancements of field at surfaces between $\mathrm{Ag}$ and $\mathrm{SiO}_{2}$ layers in some cases related with the thickness of $\mathrm{SiO}_{2}$ layers. The coupled plasmon modes were resulted by the resonance waves on four surfaces in these cases.
\end{abstract}

\section{Keywords}

Multilayered Thin-Film, Surface Plasmon, Resonance Absorption, Bi-Grating

\section{Introduction}

Periodically corrugated thin metal films have an interesting property such as the partial or total absorption of incident light energy. The absorption is associated with the excitation of the surface plasmons and is then termed the resonance absorption [1] [2] [3]. Most of studies on the resonance absorption have mainly dealt with a thin metal film grating whose surfaces are periodic in one direction [4] [5] [6].

In our previous study, we examined the excitation of coupled plasmon modes 
in a thin-film grating made of a metal [7]. When the metal is thick, e.g., more than ten times the skin depth, the plasmon can be excited on the lit surface alone. This is termed a single-interface surface plasmon (SISP). When the thickness is decreased, the plasmon can be seen also on the other surface of the film. The two plasmon waves interact with each other to form two coupled plasmon modes called short-range and long-range surface plasmon (SRSP and LRSP) [8].

In the present research, we consider a sandwiched structure:

metal/dielectric/metal, which is interesting for the application in development of optical equipment, for example improving sensitivity of clinical sensing [9], surface enhanced phenomena such as Ramman scattering, and solar cells.

\section{Formulation and Method of Solution}

In this section we first formulate the problem of diffraction by multilayered thin-film bi-grating shown in Figure 1(a). After formulating the problem, we state a method of solution based on a modal-expansion approach.

\subsection{Incident Wave}

The electric and magnetic field of an incident light is given by

$$
\left[\begin{array}{c}
\boldsymbol{E}^{\mathrm{i}} \\
\boldsymbol{H}^{\mathrm{i}}
\end{array}\right](\mathrm{P})=\left[\begin{array}{c}
\boldsymbol{e}^{\mathrm{i}} \\
\boldsymbol{h}^{\mathrm{i}}
\end{array}\right] \exp \left(i \boldsymbol{k}^{\mathrm{i}} \cdot \boldsymbol{P}\right)
$$

where $\boldsymbol{e}^{\mathrm{i}}$ and $\boldsymbol{h}^{\mathrm{i}}$ are the electric- and magnetic-field amplitude; $\boldsymbol{k}^{\mathrm{i}}=[\alpha, \beta,-\gamma]$ is the incident wave vector with $\alpha=n_{0} k^{\mathrm{i}} \sin \theta \cos \varphi$, $\beta=n_{0} k^{\mathrm{i}} \sin \theta \sin \varphi, \quad \gamma=n_{0} k^{\mathrm{i}} \cos \theta, \quad k^{\mathrm{i}}=2 \pi / \lambda$ and $n_{0}$ is the relative refractive index of region $\mathrm{V}_{0} ; \mathrm{P}=(X, Y, Z)$ is an observation point; $\lambda$ is the wavelength of the incident wave; $\theta$ is the incident angle between the $Z$-axis and the incident wave-vector; $\varphi$ is the azimuth angle between the $X$-axis and the plane of incidence.

The amplitude of the incident electric field can be decomposed into TE(TM-) component, which means the electric (or magnetic) field is perpendicular to the plane of incidence. To do this, we define two unit vectors $\boldsymbol{e}^{\mathrm{TE}}$ and $\boldsymbol{e}^{\mathrm{TM}}$ that span a plane orthogonal to $\boldsymbol{k}^{i}$. Hence, the amplitude $\boldsymbol{e}^{\mathrm{i}}$ in (1) is decomposed as

$$
\boldsymbol{e}^{\mathrm{i}}=\boldsymbol{e}^{\mathrm{TE}} \cos \delta+\boldsymbol{e}^{\mathrm{TM}} \sin \delta
$$

where the symbol $\delta$ is the polarization angle between $\boldsymbol{e}^{\mathrm{i}}$ and $\boldsymbol{e}^{\mathrm{TE}}$ shown in Figure 1(b).

\subsection{Diffracted Wave}

We seek for the diffracted fields $E_{l}(\mathrm{P})$ and $H_{l}(\mathrm{P})$ in each region. These should satisfy the following requirements.

(C1) The Helmholtz equations in each region;

(C2) Radiation conditions: The diffracted waves in $\mathrm{V}_{0}$ (or $\mathrm{V}_{L}$ ) should propagate or attenuate in the positive (or negative) $Z$-direction; 


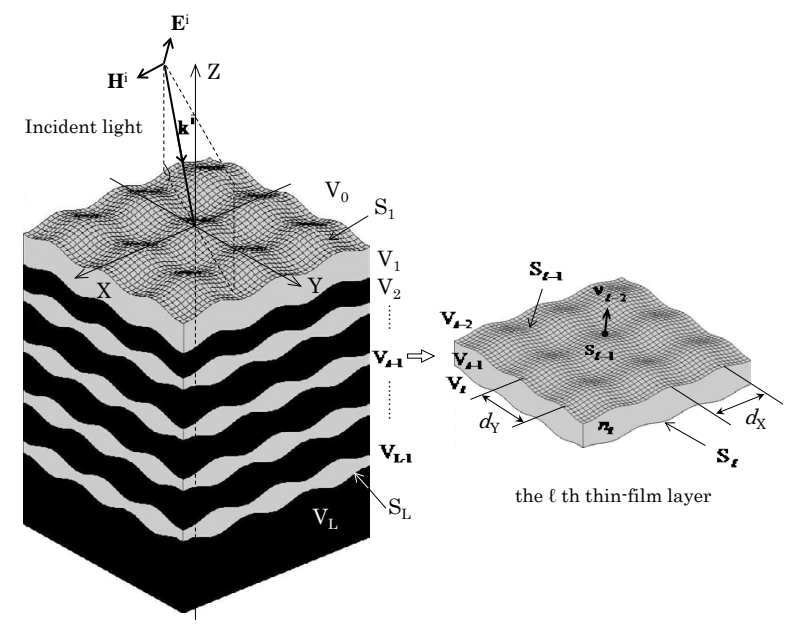

(a)

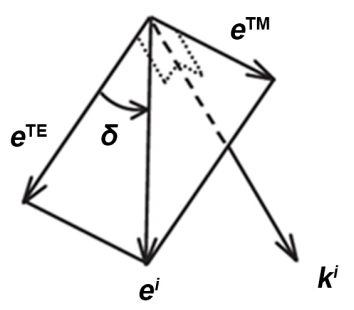

(b)

Figure 1. (a) Schematic representation of multilayered thin-film bi-grating with an incident light $\left(\mathrm{V}_{0}:\left\{\mathrm{P} \mid Z>\mathrm{S}_{1}(X, Y)\right\}, \mathrm{V}_{\mathrm{L}}:\left\{\mathrm{P} \mid Z<\mathrm{S}_{L}(X, Y)\right\}\right)$; (b) Definition of a polarization angle.

(C3) Periodicity conditions: Each component of the diffracted electric and magnetic field should satisfy

$$
\begin{gathered}
f(X+d, Y, Z)=\exp (i \alpha d) f(X, Y, Z), \\
f(X, Y+d, Z)=\exp (i \beta d) f(X, Y, Z)
\end{gathered}
$$

where, $\alpha$ and $\beta$ are the phase constants in $X$ and $Y$.

(C4) Boundary conditions: The tangential components of electric and magnetic fields are continuous across the boundaries $\mathrm{S}_{l}$.

\subsection{Geometry of the Multilayered Thin-Film Bi-Grating}

The multilayered thin-film bi-grating which is laminating $L-1$ grating layers has period $d$ in both $X$ - and $Y$-directions shown in Figure 1(a). The semi-infinite region over the multilayered bi-grating and the substrate are denoted by $\mathrm{V}_{0}$ and $\mathrm{V}_{L}$, respectively. Moreover, each region inside the multilayered bi-grating, which is numbered starting from the incident side, is denoted by

$\mathrm{V}_{\ell}(\ell=1,2, \cdots, L-1)$. The regions $\mathrm{V}_{\ell}(\ell=0,1, \cdots, L)$ are filled with isotropic and homogeneous media with refractive indices $n_{\ell}$, and a permeability of each region is equal with that of the vacuum $\mu_{0}$. The interface between $V_{\ell}$ and $\mathrm{V}_{\ell+1}$ is denoted by $\mathrm{S}_{\ell}(\ell=1,2, \cdots, L)$. The profile of $\mathrm{S}_{\ell}$ is sinusoidal and given by

$$
z=\eta_{\ell}(x, y)=\frac{H_{l}}{2}\left\{\cos \left(\frac{2 \pi x}{d_{x}}\right)+\cos \left(\frac{2 \pi y}{d_{y}}\right)\right\}-\sum_{i=1}^{\ell-1} \omega_{i}
$$

where $\omega_{\ell}$ denotes an average distance between $\mathrm{S}_{\ell}$ and $\mathrm{S}_{l+1}$. The value $H_{\ell}$ is regarded as a groove depth of the boundary $\mathrm{S}_{\ell}$ 


\subsection{Method of Solution}

We solve the problem above using Yasuura's method of modal expansion [10]. To do this, we first define the set of modal functions; next we construct approximate solutions in terms of finite modal expansions with unknown coefficients; and, finally we determine the coefficients applying the boundary conditions.

Modal functions: Because the diffracted waves have both TE- and TM-components, we need TE and TM vector modal functions in constructing the solutions. Here we employ the functions derived from the Floquet modes (separated solutions of the Helmholtz equations satisfying the periodicity (C3) and the radiation conditions (C2) if necessary). The modal functions for electric fields for each region are given by

$$
\boldsymbol{\varphi}_{\ell m n}^{\mathrm{TE}, \mathrm{TM} \pm}(\mathrm{P})=\boldsymbol{e}_{\ell m n}^{\mathrm{TE}, \mathrm{TM} \pm} \exp \left(i \boldsymbol{k}^{\mathrm{i}} \cdot \boldsymbol{P}\right)
$$

where, $m, n=0, \pm 1, \pm 2, \cdots$ and $l=0,1,2, \cdots, L$, and wave vectors in (6) are defined by

$$
\boldsymbol{e}_{\ell m n}^{\mathrm{TE} \pm}=\frac{\boldsymbol{k}_{\ell m n}^{ \pm} \times \boldsymbol{i}_{Z}}{\left|\boldsymbol{k}_{\ell m n}^{ \pm} \times \boldsymbol{i}_{Z}\right|}, \boldsymbol{e}_{\ell m n}^{\mathrm{TM} \pm}=\frac{\boldsymbol{e}_{\ell m n}^{\mathrm{TE} \pm} \times \boldsymbol{k}_{\ell m n}^{ \pm}}{\left|\boldsymbol{e}_{\ell m n}^{\mathrm{TE} \pm} \times \boldsymbol{k}_{\ell m n}^{ \pm}\right|}
$$

and

$$
\begin{aligned}
& \boldsymbol{k}_{\ell m n}^{ \pm}=\left[\alpha_{m}, \beta_{n}, \pm \gamma_{\ell m n}\right], \alpha_{m}=\alpha+\frac{2 m \pi}{d_{x}}, \beta_{n}=\beta+\frac{2 n \pi}{d_{y}}, \\
& \gamma_{\ell m n}=\left(n_{\ell}^{2} k^{2}-\alpha_{m}^{2}-\beta_{n}^{2}\right)^{1 / 2}
\end{aligned}
$$

where $\operatorname{Re}\left(\gamma_{\ell m n}\right) \geq 0$ and $\operatorname{Im}\left(\gamma_{\ell m n}\right) \geq 0$. We use the modal functions defined in equations from (6) to (8) to construct approximations of diffracted electric fields. For the accompanying magnetic fields, we employ

$$
\boldsymbol{\psi}_{\ell m n}^{\mathrm{TE}, \mathrm{TM} \pm}(\mathrm{P})=\frac{1}{\omega \mu_{0}} \boldsymbol{k}_{\ell m n}^{ \pm} \times \boldsymbol{\varphi}_{\ell m n}^{\mathrm{TE}, \mathrm{TM} \pm}
$$

Approximate solutions: To satisfy the radiation condition (C2), the approximate solution in $\mathrm{V}_{0}$ should have a form of finite linear combination of up-going modal functions with unknown coefficients. Likewise, the solution in $\mathrm{V}_{L}$ must be a linear combination of down-going modal functions. The solution in $V_{b}$, however, must have both up- and down-going waves. To show the traveling direction of a modal function, we use superscripts + and - representing up- and down-going waves. Here, we form approximate solutions for the diffracted electric and magnetic fields in $\mathrm{V}_{\boldsymbol{l}}$.

$$
\begin{aligned}
& \left(\begin{array}{c}
\boldsymbol{E}_{\ell N}^{\mathrm{d}} \\
\boldsymbol{H}_{\ell N}^{\mathrm{d}}
\end{array}\right)(\mathrm{P})=\sum_{m, n=-N}^{N} A_{\ell m n}^{\mathrm{TE}+}(N)\left(\begin{array}{l}
\phi_{\ell m n}^{\mathrm{TE}+} \\
\psi_{\ell m n}^{\mathrm{TE}+}
\end{array}\right)(\mathrm{P})+\sum_{m, n=-N}^{N} A_{\ell m n}^{\mathrm{TM}+}(N)\left(\begin{array}{l}
\phi_{\ell m n}^{\mathrm{TM}+} \\
\psi_{\ell m n}^{\mathrm{TM}+}
\end{array}\right)(\mathrm{P}) \\
& +\sum_{m, n=-N}^{N} A_{\ell m n}^{\mathrm{TE}-}(N)\left(\begin{array}{l}
\phi_{\ell m n}^{\mathrm{TE}-} \\
\psi_{\ell m n}^{\mathrm{TE}-}
\end{array}\right)(\mathrm{P})+\sum_{m, n=-N}^{N} A_{\ell m n}^{\mathrm{TM}-}(N)\left(\begin{array}{l}
\phi_{\ell m n}^{\mathrm{TM}-} \\
\psi_{\ell m n}^{\mathrm{TM}-}
\end{array}\right)(\mathrm{P}),(\ell=1,2, \cdots, L)
\end{aligned}
$$

where $N$ denotes the truncation number.

Boundary matching: Because the approximate solutions satisfy the require- 
ments (C1), (C2), and (C3) by definition, the unknown coefficients $A_{\ell m n}^{\mathrm{TE} \pm}(N)$ and $A_{l m n}^{\mathrm{TM} \pm}(N)$ are determined such that the solutions satisfy the boundary conditions (C4) in an approximate sense. In the Yasuura's method, the least-squares method is employed to fit the solution to the boundary conditions [10] [11]. That is, we find the coefficients that minimize the weighted mean-square error by

$$
\begin{aligned}
I_{N}= & \int_{S_{1}^{\prime}}\left|\boldsymbol{v} \times\left[\boldsymbol{E}_{1 N}^{\mathrm{d}}+\boldsymbol{E}^{\mathrm{i}}-\boldsymbol{E}_{2 N}^{\mathrm{d}}\right]\left(s_{1}\right)\right|^{2} \mathrm{~d} s+\left|\Gamma_{1}\right|^{2} \int_{S_{1}^{\prime}}\left|\boldsymbol{v} \times\left[\boldsymbol{H}_{1 N}^{\mathrm{d}}+\boldsymbol{H}^{\mathrm{i}}-\boldsymbol{H}_{2 N}^{\mathrm{d}}\right]\left(s_{1}\right)\right|^{2} \mathrm{~d} s \\
& +\sum_{l=2}^{L-1}\left\{\int_{S_{l}^{\prime}}\left|\boldsymbol{v} \times\left[\boldsymbol{E}_{l N}^{\mathrm{d}}-\boldsymbol{E}_{l+1 N}^{\mathrm{d}}\right]\left(s_{l}\right)\right|^{2} \mathrm{~d} s+\left|\Gamma_{l}\right|^{2} \int_{S_{l}^{\prime}}\left|\boldsymbol{v} \times\left[\boldsymbol{H}_{l N}^{\mathrm{d}}-\boldsymbol{H}_{l+1 N}^{\mathrm{d}}\right]\left(s_{l}\right)\right|^{2} \mathrm{~d} s\right\}
\end{aligned}
$$

where $S_{1}^{\prime}$ denotes one-period cells of the interface $S_{\ell}, \Gamma_{\ell}$ is the intrinsic impedance of the medium in $\mathrm{V}_{\ell}$ and $\boldsymbol{v}$ is a unit normal vector of each boundary.

To solve the least-squares problem on a computer, we need a discretized form of the problem. We first discretize the weighted mean-square error $I_{N}$ by applying a two-dimensional trapezoidal rule where the number of sampling points is chosen as $2(2 N+1)$ [9]. We then employ orthogonal decomposition methods [singular-value decomposition (SVD) and QR decomposition (QRD)] in solving the discretized problem [12] [13].

It is known that the solutions obtained by Yasuura's method have proof of convergence [13] [14]. We, therefore, can employ the coefficients $A_{\ell m n}^{\mathrm{TE} \pm}(N)$ and $A_{\ell m n}^{\mathrm{TM} \pm}(N)$ with sufficiently large $N$ for which the coefficients are stable in evaluating diffracted fields. The power reflection and transmission coefficient of the $(m, n)$ order propagating mode in $\mathrm{V}_{0}$ and $\mathrm{V}_{L}$ are given by

$$
\begin{gathered}
R_{m n}=\frac{\gamma_{0 m n}}{\gamma}\left|A_{1 m n}^{\mathrm{TE}+}\right|^{2}+\frac{\gamma_{0 m n}}{\gamma}\left|A_{1 m n}^{\mathrm{TM}+}\right|^{2}, \operatorname{Re}\left(\gamma_{0 m n}\right) \geq 0, \\
T_{m n}=\frac{\gamma_{L m n}}{\gamma}\left|A_{L m n}^{\mathrm{TE}-}\right|^{2}+\frac{\gamma_{L m n}}{\gamma}\left|A_{L m n}^{\mathrm{TM}-}\right|^{2}, \operatorname{Re}\left(\gamma_{L m n}\right) \geq 0 .
\end{gathered}
$$

The coefficient defined above is the power carried away by propagating diffraction orders normalized by the incident power.

\section{Numerical Results}

The multilayered bi-grating is made by 3 layers: $/ \mathrm{Ag} / \mathrm{SiO}_{2} / \mathrm{Ag} /$. The incident light is a TM-polarized plane wave with a $650 \mathrm{~nm}$ wavelength. The relative refractive index of Vacuum $=1, n_{\mathrm{Ag}}=0.07+4.2 i$ and $n_{\mathrm{SiO}_{2}}=1.5$. The periods of two directions $d_{\mathrm{x}}=d_{\mathrm{y}}=556 \mathrm{~nm}$. We consider 3 types of gratings with different thickness pairs of each region: (A) $e_{\mathrm{Ag}}=e_{\mathrm{SiO}_{2}}=27.8 \mathrm{~nm}$; (B) $e_{\mathrm{Ag}}=27.8 \mathrm{~nm}, e_{\mathrm{SiO}_{2}}=278$ $\mathrm{nm}$; and $(\mathrm{C}) e_{\mathrm{Ag}}=278 \mathrm{~nm}, e_{\mathrm{SiO}_{2}}=27.8 \mathrm{~nm}$. We will then calculate the diffraction efficiencies and field distributions of these gratings.

Figure 2 shows the $(0,0)$-th order reflection and transmission coefficient as functions of the incident angle $\theta$ for 3 types of gratings with the azimuth angle $\phi=45^{\circ}$. Five dips are observed on reflection curves throughout Figures 2 (a)-(c), the field distributions of the total electric fields in the vicinity of the $\mathrm{SiO}_{2}$ layer for these dips are shown in Figures 3(a)-(e). Distances in the $Z$ direction are normalized by the wavelength. 


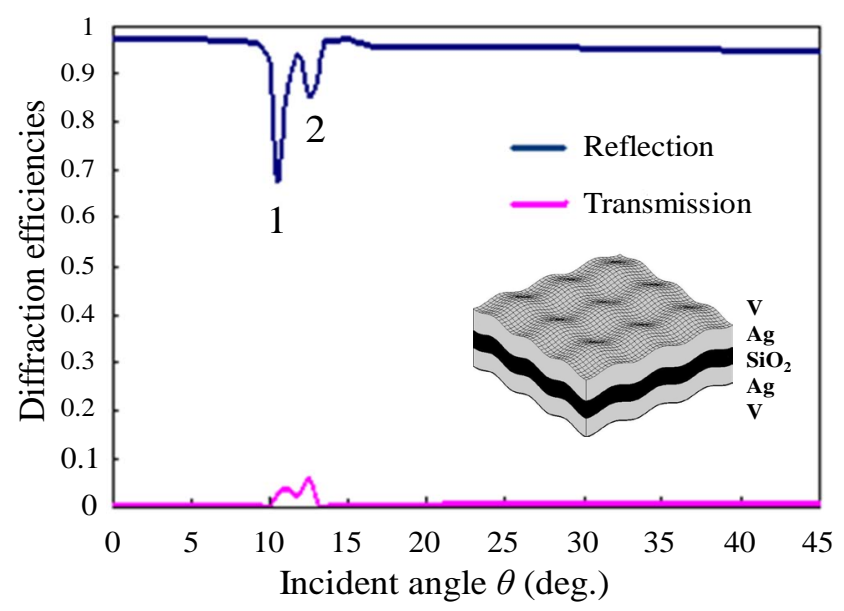

(a) Type a

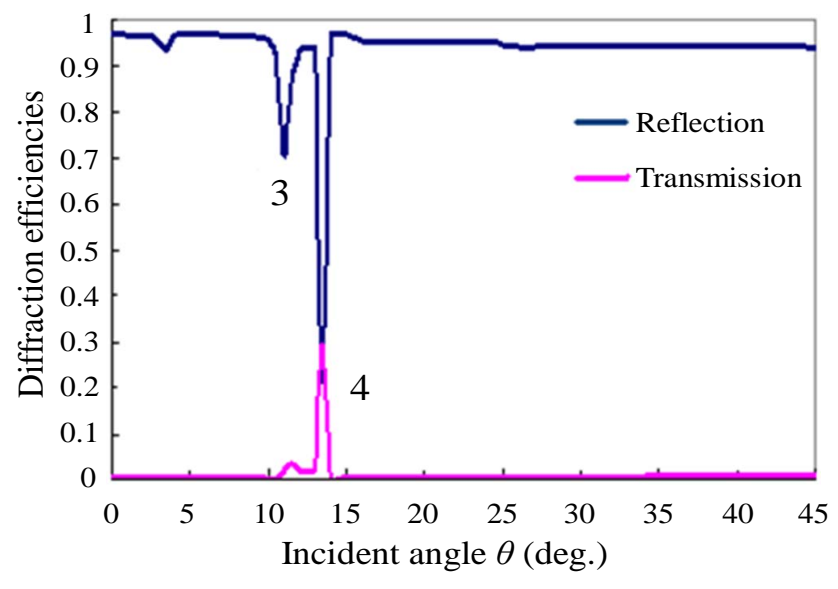

(b) Type b

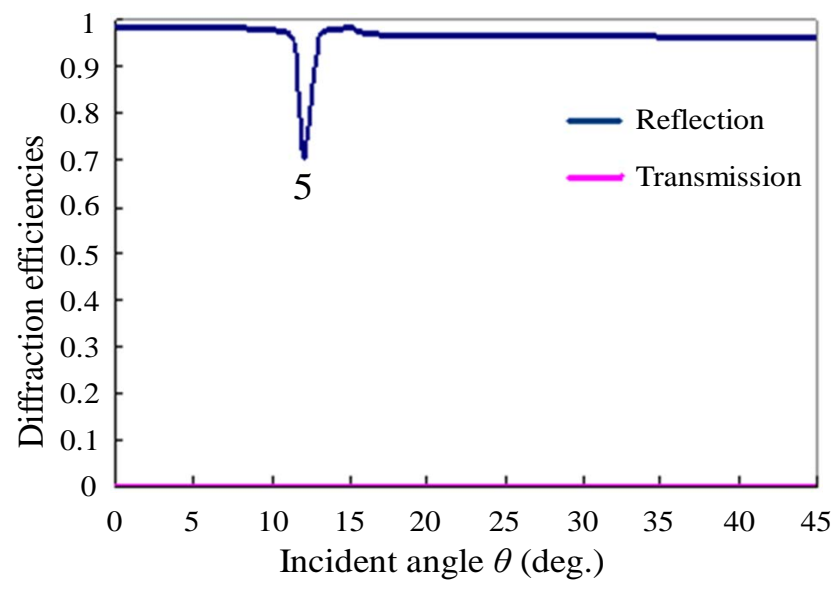

(c) Type c

Figure 2. The $(0,0)$-th order reflection and transmission coefficient for 3-layers doubly periodic gratings with 3 types of thickness pairs.

For type A shown in Figure 2(a), two dips (dip 1 at $\theta=106^{\circ}$ and dip 2 at $\theta=$ $12.5^{\circ}$ ) are observed on reflection curve and the transmission coefficient also increases at the same time. Figures 3(a) and Figures 3(b) show the field distributions for dips1 and 2. Strong field enhancements are observed at each surface between Vacuum and Ag layers for either of the two dips. The interaction of SPRs excited on these surfaces result in the coupled plasmon modes (SRSP or LRSP).

For type B shown in Figure 2(b), two dips (dip 3 at $\theta=11.0^{\circ}$ and $\operatorname{dip} 4$ at $\theta=$ $13.5^{\circ}$ ) are observed on reflection curve and the transmission coefficient increases at the same time. Reflection at dip 4 is much lower than that of dips 1 and 2 accompanying change of the thickness of $\mathrm{SiO}_{2}$. Figure 3(c) and Figure 3(d) show the field distributions for dips 3 and 4. Strong field enhancements are observed at each surface between Vacuum and Ag layers, similar to that of type A. In addition, fields enhance strongly at two surfaces between $\mathrm{Ag}$ and $\mathrm{SiO}_{2}$ for dip 4 at the same time. This means coupled plasmon modes are resulted by resonance waves excited at four surfaces. 


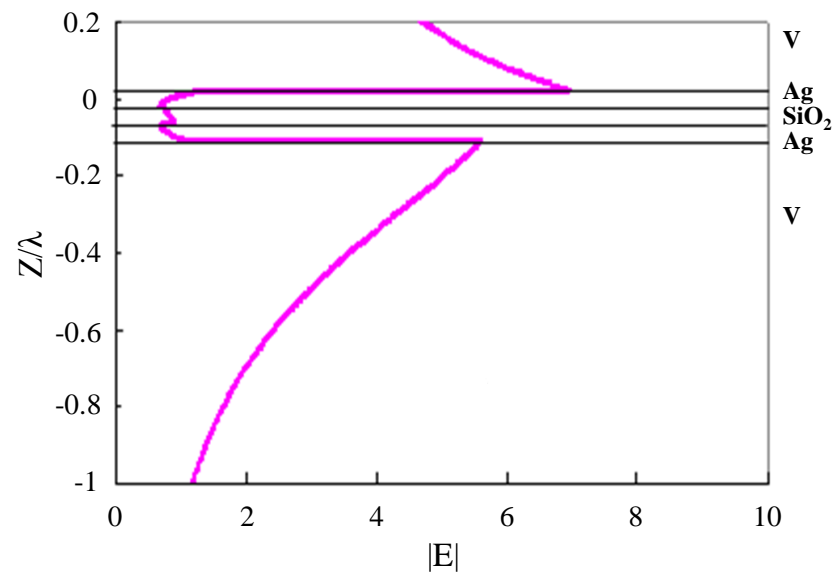

(a) Dip 1

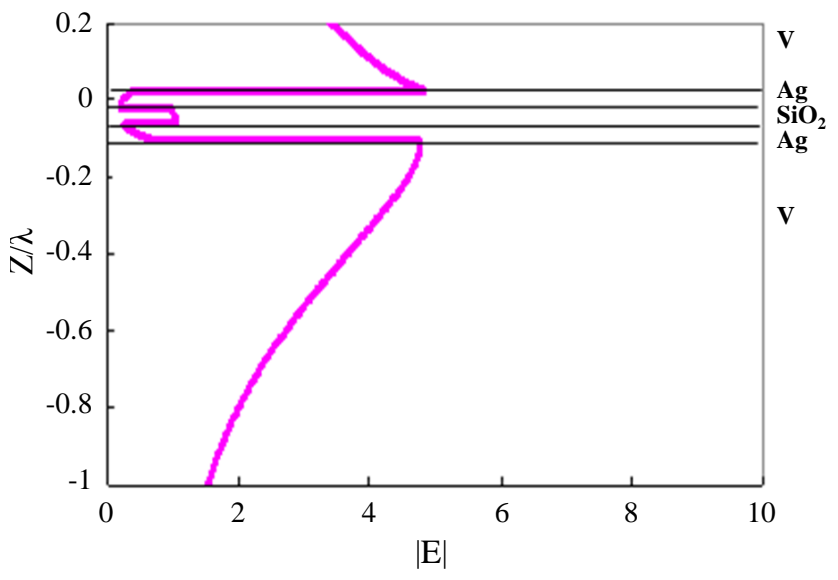

(b) Dip 2

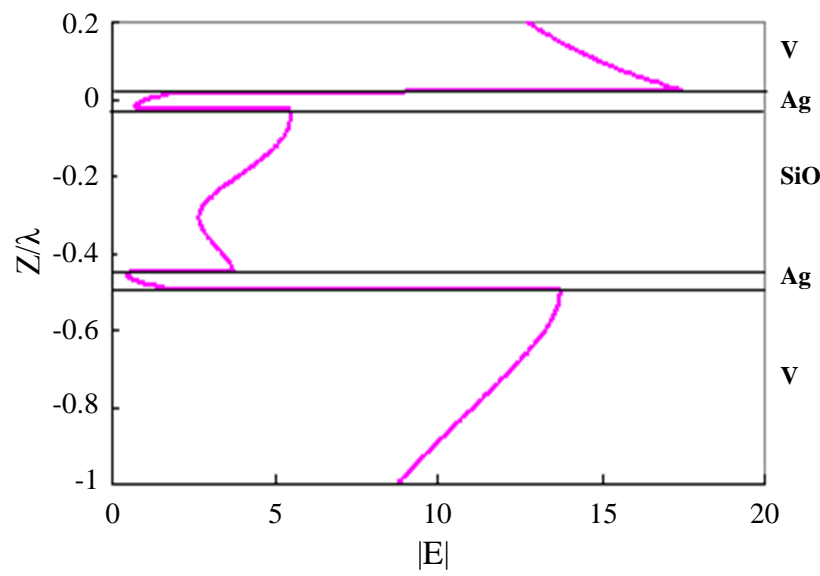

(d) Dip 4

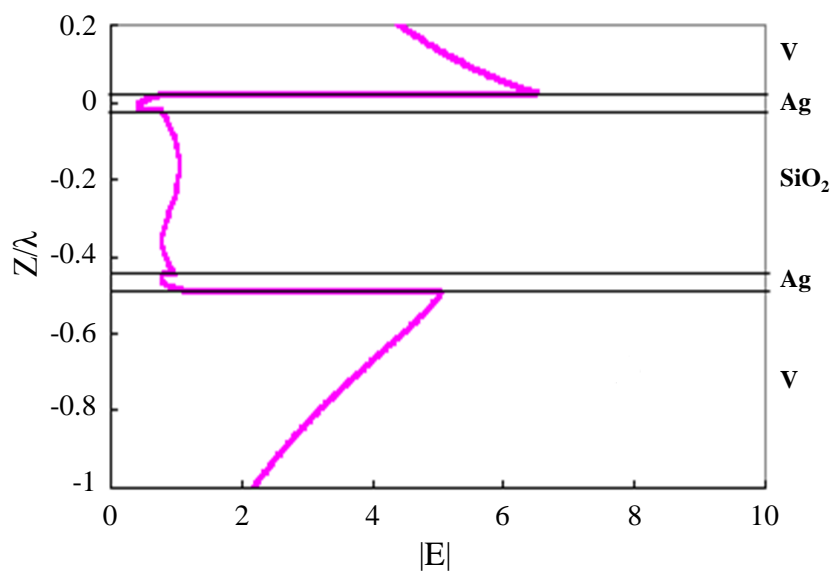

(c) Dip 3

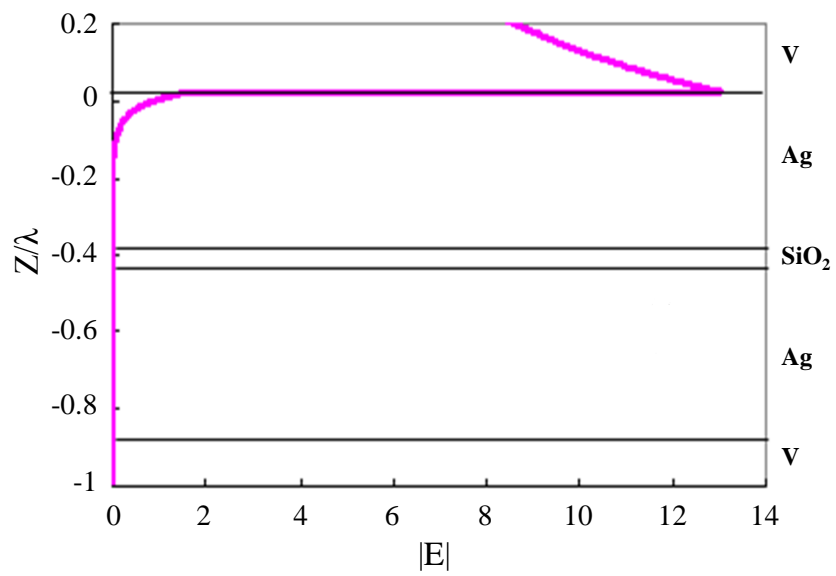

(e) Dip 5

Figure 3. Field distributions correspond to five dips.

For type $\mathrm{C}$ shown in Figure 2(c), one dip (dip 5 at $\theta=12.0^{\circ}$ ) is observed on reflection curve and the transmission coefficient keeps to zero. Figure 3(e) shows the field distribution for dip 5. Field enhanced only on the lit surface and quickly attenuates through the $1^{\text {st }} \mathrm{Ag}$ layer as shown in Figure 3(e). Because the 
$1^{\text {st }} \mathrm{Ag}$ layer is enough thick, the oscillation near the upper surface does not reach the lower surface and, hence, the field below the $1^{\text {st }} \mathrm{Ag}$ layer is zero. This indicates the excitation of SISP.

\section{Conclusion}

We solved the problems for 3 thin-films bi-grating. By calculating the diffraction efficiency and field distributions, we showed that the SPR phenomenon excited and we observed: 1) excitation of a SISP mode at the lit surface of the $1^{\text {st }} \mathrm{Ag}$ layer with strong field enhancement for thick enough layer case; 2) excitation of coupled SPR modes (SRSP or LRSP) at each surface between vacuum and Ag layers with strong field enhancements for thin enough Ag layer cases no matter with the thickness of $\mathrm{SiO}_{2}$ layers; 3) enhancements of field at surfaces between $\mathrm{Ag}$ and $\mathrm{SiO}_{2}$ layers in some cases related with the thickness of $\mathrm{SiO}_{2}$ layers. The coupled plasmon modes were resulted by the resonance waves excited on four surfaces in these cases. In future, we plan to study applications for multilayered bi-grating such as improving the sensitivity of a bio-sensor by determining changes of SPRs excited at different layers' surfaces.

\section{Conflicts of Interest}

The author declares no conflicts of interest regarding the publication of this paper.

\section{References}

[1] Raether, H. (1977) Physics of Thin Films, 9, 145-261.

[2] Raeter, H. (1982) Surface Plasmon and Roughness. In: Agranovich, V.M. and Mills, D.L., Eds., Surface Polaritons, North-Holland, Amsterdam, 331-403. https://doi.org/10.1016/B978-0-444-86165-8.50015-3

[3] Nevier, M. (1980) The Homogenous Problem. In: Petited, R., Ed., Electromagnetic Theory of Gratings, Springer-Verlag, Berlin, 123-157. https://doi.org/10.1007/978-3-642-81500-3 5

[4] Economou, E.N. (1969) Physical Review, 182, 539-554. https://doi.org/10.1103/PhysRev.182.539

[5] Chen, Z., Hooper, I.R. and Sambles, J.R. (2008) Physical Review B, 77, Article ID: 161405. https://doi.org/10.1103/PhysRevB.77.161405

[6] Maksimenko, L., Matyash, I., Mischuk, O., Rudenko, S., Serdega, B. and Stetsenko, M. (2014) Journal of Modern Physics, 5, 617-626. https://doi.org/10.4236/jmp.2014.58072

[7] Suyama, T., Zhang, Y.J., Okuno, Y., Luo, Z.Q. and Matsuda, T. (2010) PIERS Online, 6, 76-80. https://doi.org/10.2529/PIERS090902043932

[8] Hibbins, A.P., Murray, W.A., Tyler, J., Wedge, S., Barnes, W.L. and Sambles, J.R. (2006) Physical Review B, 74, Article ID: 073408.

https://doi.org/10.1103/PhysRevB.74.073408

[9] Ahn, J.H., Seong, T.Y., Kim, W.M., Lee, T.S., Kim, I. and Lee, K.S. (2012) Optics Express, 20, 21729-21738. https://doi.org/10.1364/OE.20.021729

[10] Yasuura, K. and Itakura, T. (1966) Approximation Method for Wave Functions (I), 
(II), and (III). Kyushu Univ. Tech. Rep., Vol. 38, 72-77, 1965; Vol. 38, 378-385, 1966; Vol. 39, 51-56.

[11] Okuno, Y. (1990) Mode-Matching Method. In: Yamashita, E., Ed., Analysis Methods for Electromagnetic Wave Problems, Artech House, Boston, 107-138.

[12] Lawson, C.L. and Hanson, R.J. (1974) Solving Least Squares Problem. Prentice-Hall, Englewood Cliffs.

[13] Suyama, T., Okuno, Y., Matsushima, A. and Ohtsu, M. (2008) Progress in Electromagnetics Research B, 2, 83-102. https://doi.org/10.2528/PIERB07110301

[14] Matsuda, T. and Okuno, Y. (1996) Radio Science, 31, 1791-1798.

https://doi.org/10.1029/96RS02153 